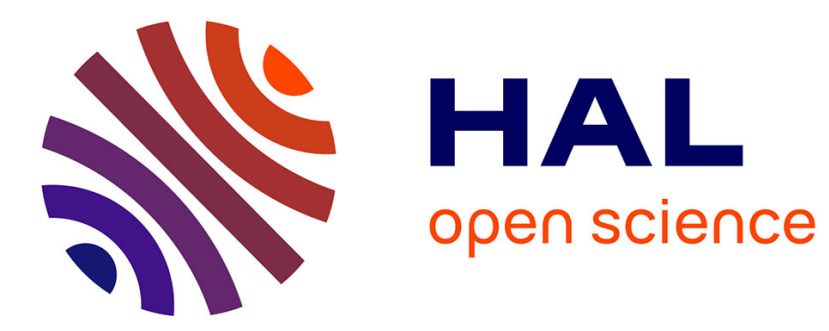

\title{
Recherches archéologiques dans la région d'Aix-en-Provence
}

\author{
Fernand Benoit
}

\section{To cite this version:}

Fernand Benoit. Recherches archéologiques dans la région d'Aix-en-Provence. Gallia - Fouilles et monuments archéologiques en France métropolitaine, 1954, 12 (2), pp.285-300. 10.3406/galia.1954.1391 . hal-01924465

\section{HAL Id: hal-01924465 \\ https://hal.science/hal-01924465}

Submitted on 3 Mar 2020

HAL is a multi-disciplinary open access archive for the deposit and dissemination of scientific research documents, whether they are published or not. The documents may come from teaching and research institutions in France or abroad, or from public or private research centers.
L'archive ouverte pluridisciplinaire HAL, est destinée au dépôt et à la diffusion de documents scientifiques de niveau recherche, publiés ou non, émanant des établissements d'enseignement et de recherche français ou étrangers, des laboratoires publics ou privés.

\section{(이) $\$$}

Distributed under a Creative Commons Attribution - NonCommercial - NoDerivatives| 4.0 


\title{
RECHERCHES ARCHÉOLOGIQUES I)ANS LA RÉGION D'AIX-EN-PROVENCE
}

\author{
par M. Fernand BeNoIT
}

\section{1. - Les fouildes d'ENTremont en 1953-1954*}

La fouille des campagnes 1953-1954 a portó sur les insulae bordant au S. el au N. la rue XVII 2 , a la limile de la pareelle 3407 oceupée par l'Aviation (fig. 1). Eille a permis de reconnaître deux niveaux, Irìs rapprochés par la date et de mettre au jour un édifice religieux en rapporl avec le "culte des crûnes ".

Le premier niveau de cette rue, à quelques centimètres au-dessous des fondalions des cases, est marqué par un petit cailloutis, tassé, anaiogue à celui de la "voie sacrée" où avait été trouvéc la statuaire (3407). A ce niveau quelques menus fragments de poterie campanienne et indigène contrastent par leur fractionnement avec la quantité de vaisselle el de dolia qui recouvrent le niveau le plus récent, daté de la destruction de 123.

La prospection des fonds de cases avec le détecteur de mines, difficile parce que celui-ci est un instrument sensible ì la céramique et au métal ef n'a pas d'aclion au delà de $40 \mathrm{~cm}$ environ ', a permis de découvrir à l'angle de la case XX 3, un silo creusé dans le gypse (prof. $1 \mathrm{~m}$; diam. $0^{\mathrm{m}} 60$ env.), dont la paroi était revêtue de plaques de calcaire cramponnées avec des clous de fer. Il paraît appartenir à la première époque de l'habitat, établi sur le lit du rocher, irrégulier, et avoir été obstrué par le comblement de terre sur lequel reposenl les murs de la case, à $0^{\mathrm{m}}, 15$ environ au-dessus du roc. Au haut du silo, masses de fer (marleau), pointes de javelol et de flèches de fer; sur le sol du premier niveau et dans le silo, cent oboles de Marseille (tête ì gauche),

(*) Cr. Gallia, V-1947, p. 81 et suiv. Assistant aux fouilles: R. Ambard.

(1) Le Prof. Canac, directeur du Centre de Recherches Scientifiques de Marseille, a mis all point un détecteur, dont la sensibilité permellura une prospection plus profonde. 


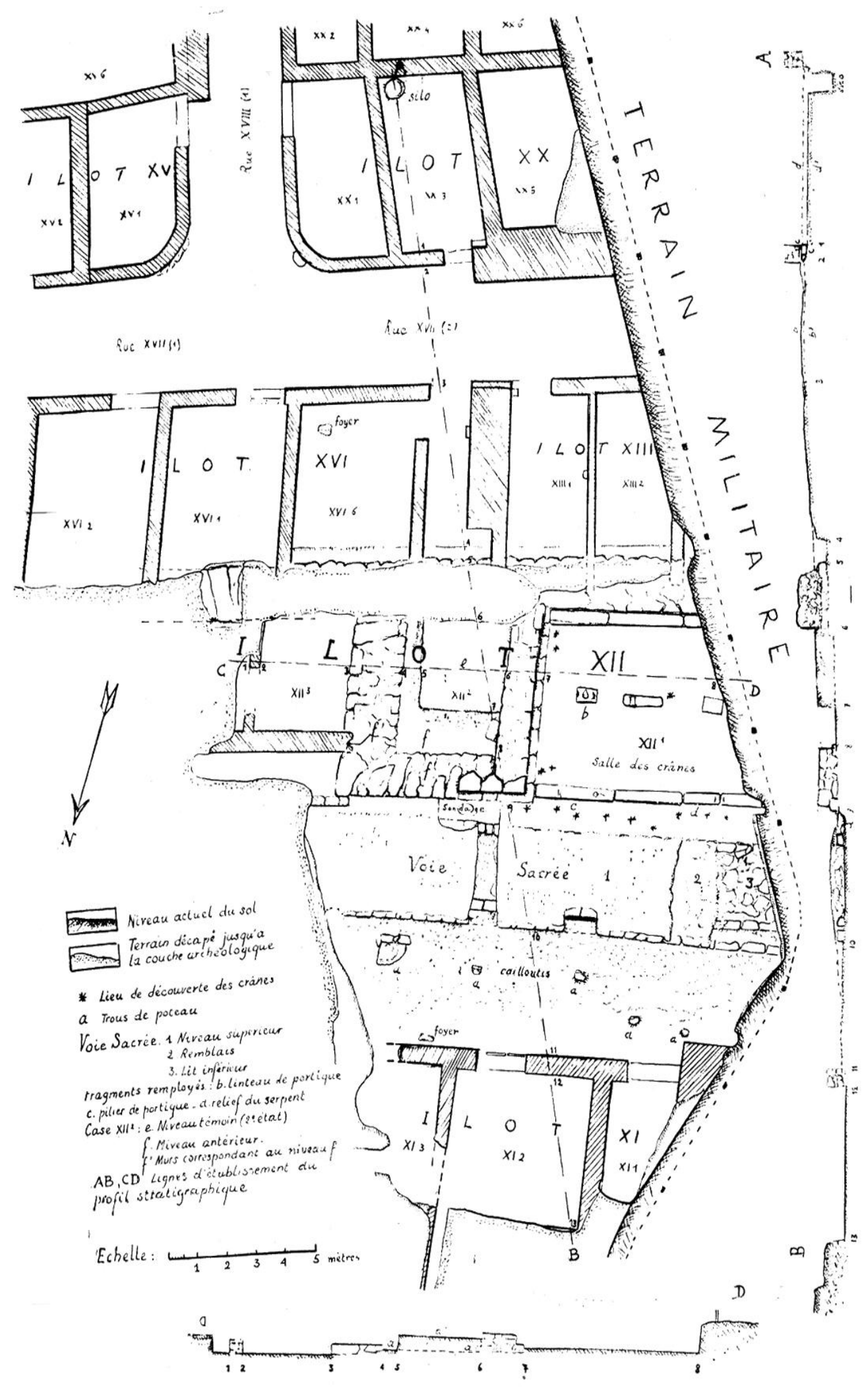

Fici. 1. - Plan el profils stratigraphiques des foullies dans le quartier du Sanchaire, suivant

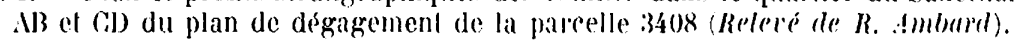

AR. 1, 2 : Mur nord de la case XX 3 avec vesliges $(c)$ du mur antéricur. $\cdots-d: 2^{\circ}$ niveau de la case XX 3 recouvrant le silo; $d^{\prime}$ : niveau anléricur de la case. - $b$ : Niveau aclucl de la rule XVII $2 ; b^{\prime}$ : niveau anlérieur. $-4,5$ : Sondage à l'exlrémité $\mathrm{N}$. de l'impasse. - 5, 6: Masse de terre et de pierre bornant au nord les cases des îlots XIII el XVI et établie sur les vestiges d'un large mur antérieur. $-6,7: 2^{\mathrm{e}}$ niveau de la case XII 2. $-7,8$ : Niveaul primilit de la casc. -- $9(a)$ : Sondage a la hase du mur bordant au S. la case XII 2 (débris d'amphores italiques, jarres, campanienne, poterie cloutée). 10, 11 (en pointilles): Nivcau de la Voie Sacrése auldessus du cailloutis anterieur.

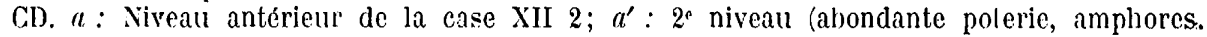
jarres, campanienne, indigine). Couche cendreuse sur loute lil superficie. 
deux drachmes ( $2 \mathrm{gr}, 5$ déc. 2 cent, et $2 \mathrm{gr}, 4$ déc. 7 cent.) au lion arqué ; trois deniers du type sans monogramme, des environs de 200 av. J.-G., intéressants pour le synchronisme du monnayage massaliète : 1 quinaire au type de la déesse Rome à droite et au revers des Dioscures, avec légende Roma et fer de lance verlical (type 240-197), ol 2 Vicloriats : lête de Jupiler et au revers Victoire couronnant un trophée, l'un avec la truic au pied de la Victoire et légende de Rome, de 240-197 av. J.-(C. (M. J. Babelon).

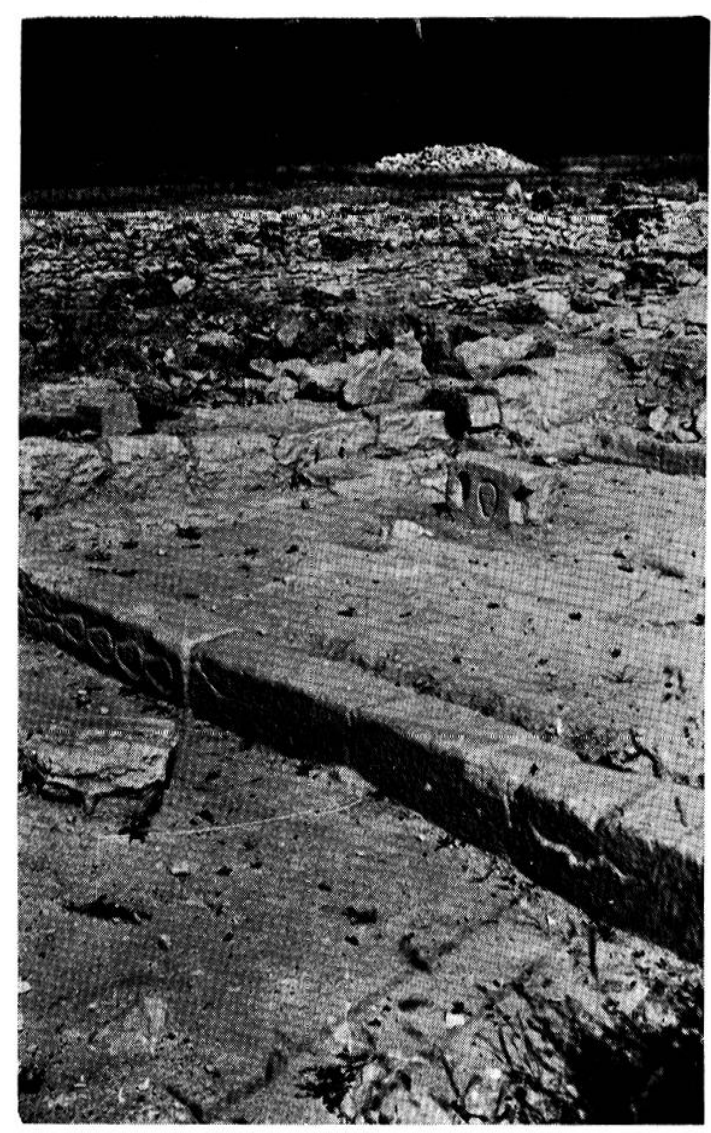

Fia. 2. -- Quartier du. sanctuaire. La vole $\left(2^{\circ} \mathrm{nI}-\right.$ veau) devant la salle lyypostyle.

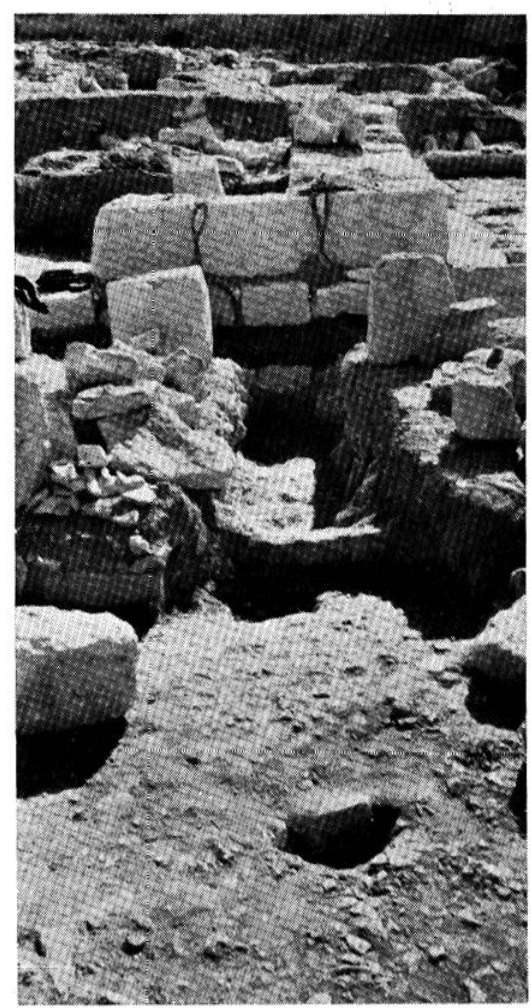

FIG. 3. - Coupe transversale de la Voie Sacrée à l'alignement du trou du poteau $a^{2}$ faisant apparaître la bordure du rudus. Au premier plan, le cailloutis du premier niveau.

Parallèlement à cette rue, est apparue vers le N. une seconde voie, en direction de la "voie sacrée" de la parcelle 3407 . Elle est bordée au S. d'édifices d'un type particulier qui révèlent une réfection antérieure à la destruction de 123 (fig. 2). La poursuite de ce dégagement vers le N.-E., permettra de préciser le plan général de ce remaniement. La rue est actuellement comprise entre deux alignements de constructions, distants d'environ 8 metres : cases 
au N.-(), datant de la première époque; salle hypostyle surélevéc au S.-E., dalant d'une réfection à une deuxième epoque. Le sol de la rue de la premiere époque, pavé de cailloutis lassé, est conservé sur une largeur de $4^{\mathrm{m}}, 10$; il présente un alignement de trous de poteaux - peut-être pour des auvents qui avaient été recouverts par l'exhaussement du niveau de ce secteur à la deuxième époque ; vers le N.-0., à proximité du mur d'une case, foyer d'argile avec cendres. - La poterie, très fragmentée, trouvée sur ce sol et celle qui a été retirée de plusieurs sondages faits en bordure de la salle hypostyle. est représentće par la campanienne à argile rose el la poterie à paroi fiue, à décoration pointillíe ou cloutié, remontant au milieu du $\mathrm{II}^{\mathrm{e}}$ siècle av. J.-C..

$\Lambda$ une seconde époque, très proche de la première, le sol a été exhaussé el mis au nivenu de celui de la rue XVII, lígèrement surélevé par suile de la déclivilé naturelle vers le N.; cette transformation du quartier a fait dispar'ấtre les construclions qui bordaient le còté $\mathrm{S}$. de la rue. Le sous-sol de la moitié S. de celle-ci, bordée de deux alignements de pierres, était constilué par un véritable rudus, composé de fragments de pierre calcaire, ayant des traces de taille, parmi lesquels un fragment de staluaire, présentant sur une face, le départ d'un cercle en relief, peut-être un clipeus, avec deux bandes en retrait (dimension de la partie aplanie de la pierre seulptée : long. 0,36; larg. 0,31); ces blocs de pierre, disposés sans ordre et parfois de champ, mélangés à des fragments de dolia, appartiennent à des ídifices de grande proportion, détruits et utilisés comme matériau (fig. 3 et 4).

Les constructions qui bordent cette voie, au Sud, présentent égulement le remploi de blocs en grand appareil, fractionnés, et un alignement de piliers légèrement pyramidaux, uppartenant à un portique, dont deux présentent des sculptures sur la tranche regardant la rue :

de l'E. à l'O., pilier de pierre calcaire, brisé au milieu (long. 2,58; larg. (face sculptée) 0,37/34 (fig. 2, à g. et fig. 5) ; ép. 0,46), olné de 12 "têtes coupées " piriformes, traitées en champlevé, de façon stylisée, les yeux marqués par une incision horizontale qui rejoint celle du nez triédrique; la bouche n'est pas indiquée; au bas de la pyramide, tête inversée ;

pilier de pierre de Bibemus, de coulcur jaunâtre (carrière d'Aix), (long. $2^{\text {m }}, 32$; larg. $0,40 / 33$; ép. $\left.0,43 / 39\right)$;

pilier de pierre calcaire (long. $1^{\mathrm{m}}, 24$; larg. (face sculplíe) $0^{\mathrm{m}}, 47$; ́́p. $\left.0^{\mathrm{m}}, 47 / 40\right)$, orné d'un signe serpentiforme, d'une ondulation sieche (long. $0^{\mathrm{m}}, 65$ ), sans doule un serpent dont la tête triangulaire est écailléc (fig. 2, à dr.) ;

pilier de pierre calcaire (long. $1^{\mathrm{m}}, 48 ;$ larg. $0^{\mathrm{m}}, 45$; ép. $\left.0^{\mathrm{m}}, 41\right)$. 

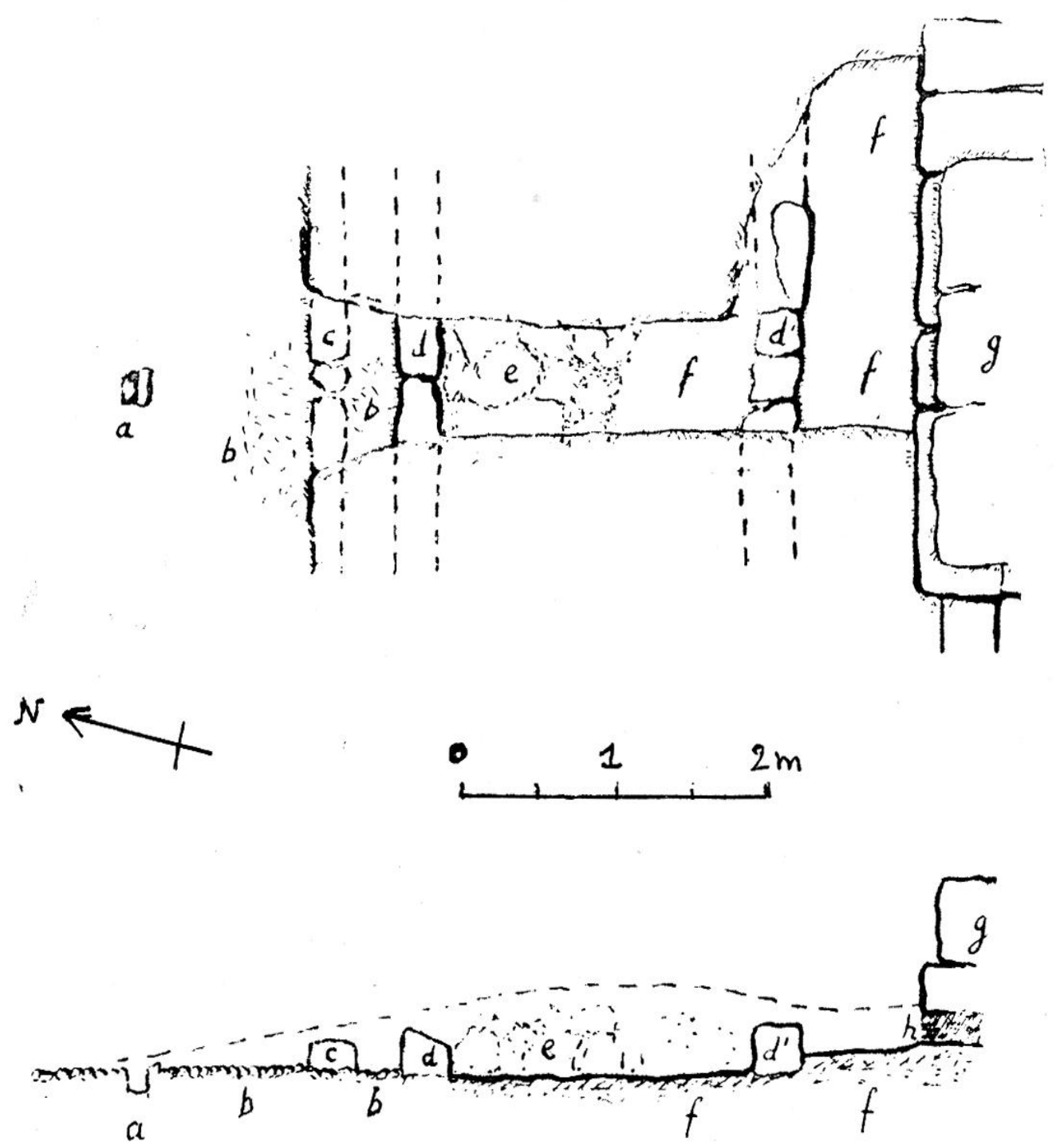

FIG. 4. - Entremonl. Sondage de la voie du sancluaire; en haul, plan; en bas, coupe. $a$ : Trou de poteau ( $a 2$ du plan d'cnsemble). $-b$ : Cailloutis. - $c:$ Chaîne de pierres bordant au N. le rudus de la Voie Sacrée établi sur le cailloulis $b .-d$ : Deuxic̀me bordure antérieure à la bordure $c .-d^{\prime}$ : Ghaîne de pierres limitant au S. lc rudus de la Voie Sacrée. - $e$ : rudus de la Voie Sacrée. - $f:$ Sol rocheux. - $g:$ Mur N. de la case XII 2. $-h$ : (fig. 2) Remblai établi sur le sol rocheux $f$ et soutenant le mur $g$. L'espace compris entre $d$ et $d^{\prime}$ est remblayé avec des pierres provenant de construrtions antérieures. L'espace compris entre $d$ et le mur $q$, contient des débris do poterie (amphores à vin italiques, indigène, fragments de potcrie cloutée).

Sur le plan, à droite : en haut salle XII 2 , en bas salle XII 1 (salle des crânes).

Cet alignement, d'une longueur totale $\mathrm{de}^{\mathrm{m}}, 62$, se prolonge vers le S.-0., dans le terrain militaire (3407).

La tranche supérieure des trois premiers bloes présente des traces d'utilisalion; sur le premier, entaille correspondant à l'assise d'un pilier de bois ou 


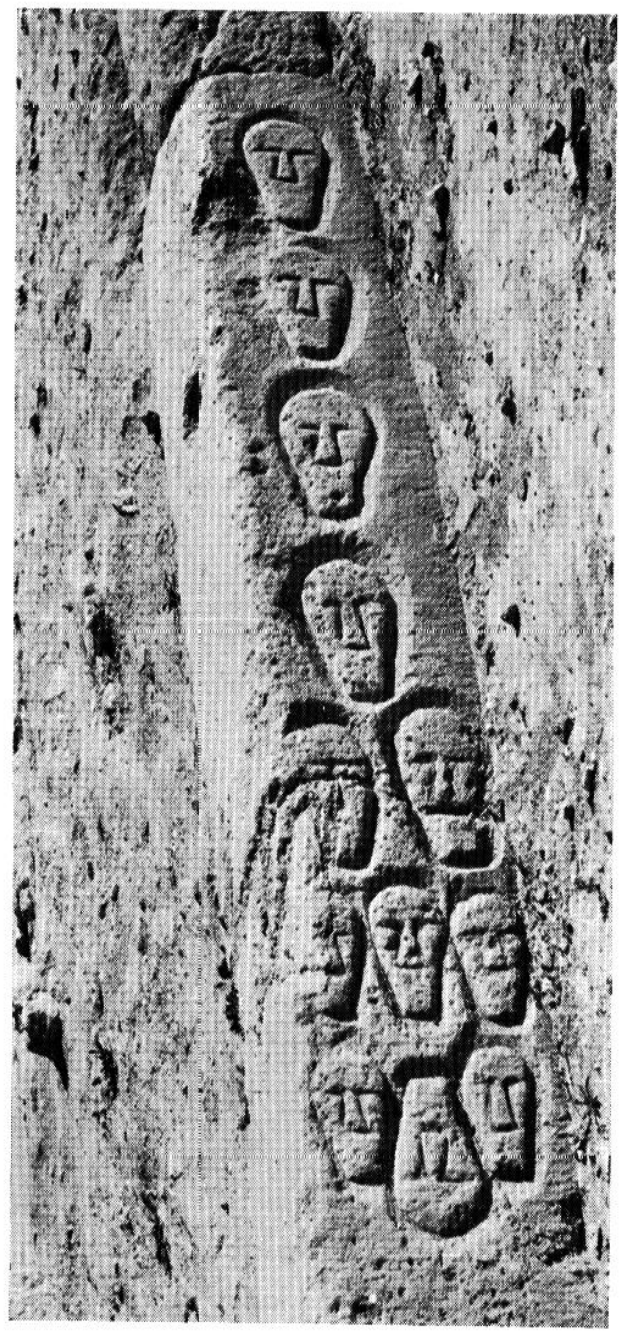

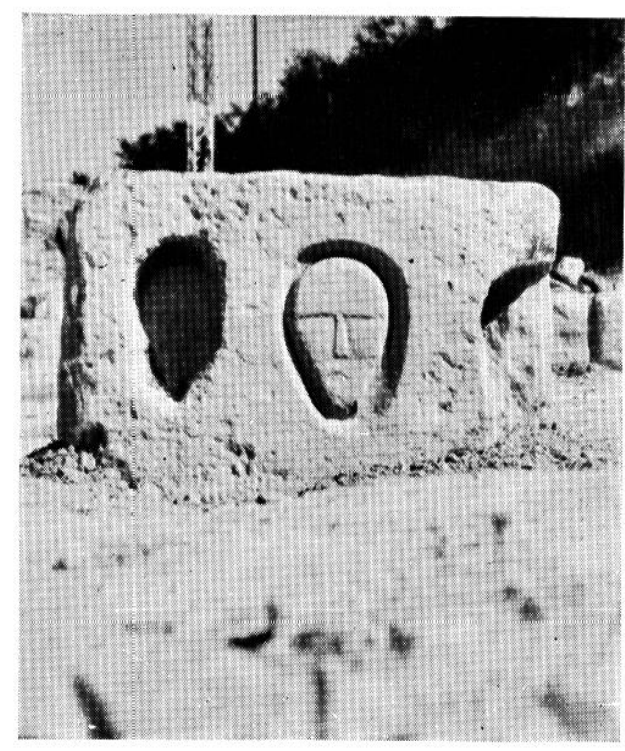

Frci. 6. - Fragment de linteau creusé d'alvéoles céphaliformes sur les deux faces. (La face décorće d'une «têle coupée » était tournée vers le haul, dans sa posilion de remploi). Les têtes sont représentées sans l'indicalion de la bouche.

FIG. 5. - Pilicr décoré de douze "tôtes coupées », dans sa position de remploi.

(Voir fig. 2. p. 28i).

d'une porte it laquelle correspondrait ì $1^{\mathrm{m}}, 25$, une profonde cupule (diam.

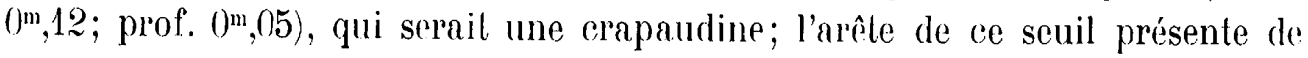
l'usure; l'extrémilé opposée des 2 blocs suivants porte nettement les traces d'une entaille rectangulaire de $0^{\mathrm{m}}, 36$ de longueur, pour l'assise d'un pilier ì la jonction des deux blocs du stylobate.

La tranche supéricure de ces piliers, élevée au-dessus du sol de la rue de la deuxième époque, de $0^{\mathrm{m}}, 30$ environ, correspond au niveau d'une salle spacieuse, large de $5^{\mathrm{m}}, 02$ (mesures intérieures) et dont la longueur jelle dépasse $8 \mathrm{~m}$ ) ne peut être connue. Vers le milieu, apparaîl un alignement de dalles, dont les deux premières émergent au niveau du sol, probablement supports de poleaux : dalle rectangulaire à l'O. en calcaire, pilier en pierre de Bibemus (long. $1^{\mathrm{m}}, 32$; larg. $0^{\mathrm{m}}, 35$ ), et au-dessus du sol, vers l'E., fragment de linteau, dont la face sculptée était tournée vers le ciel (long. $0^{\mathrm{m}}, 72$; larg. $0^{\mathrm{m}} 43$; ép. 
$\left.0^{\mathrm{m}}, 36\right)$. Cette face est ornée, au centre, d'une tête coupée (fig. 6), sans bouche, dílouríe par une gorge (haul. $0^{\mathrm{m}}, 255$ ), d'un lype analogue à celle du pilier ; clle est cncadrée de deux enlailles céphaloüdes, piriformes, dont l'une à droite, brisće par la cassure du linteau (dimensions: haut. $0^{\mathrm{m}}, 21$ et $0^{\mathrm{m}}, 22 ;$ prof. $0^{\mathrm{m}}, 06$ et $\left.0^{\mathrm{m}}, 11\right)$; la face postérieure porte deux entailles analogues, l'une inachevée (haut. $0^{\mathrm{m}}, 18$; prof. max. $\left.0^{\mathrm{m}}, 05\right)$ et l'autre lerminíc par une coupure droile vers le haut (haut. $0^{\mathrm{m}}, 18$; prof. $\left.0^{\mathrm{m}}, 11\right)$. En outre, la parlie haute de la face sculptéc et l'extrémilé de la tranche supérieure du linteau portent des incisions linćaires figurant des raies parallìles très usées pour la première, et pour la seconde, un double encadrement enfermant des losanges, dont la technique rappelle cerle des "scapulaires" des "dieux acoroupis" de Roquepertuse.

$\Lambda$ des niveaux différents, et an position inclinéce, ont étó recurillis d'imporlants fragments d'opus signinum, d'argile lassée, friable, décorés d'alignements de petils eubes de pierre caleaire blanche et de pierre noire (irréguliers de $0^{\mathrm{m}}, 005$ à $\left(0^{\mathrm{m}}, 01\right.$ de côté). Bouleversement des travaux agricoles ou écroulement de l'élage de l'édifice ? La composition de ce béton, différent de ceux des villae gréco-romaines d'Ampurias, dalís de l'ípoque de César, permet de lui donner une date antéricure a $123^{2}$, que confirment la céramique el la deslinalion de la salle hypostyle. Sur le sol de la salle el de la rue de deuxième époque, fragments de fer de pilum, boulels de basalle $\left(\right.$ diam. $\left(0^{\mathrm{m}}, 15 / 20\right)$, pelits bronzes massalides au taureau et très nombremx fragments d'amphores appartenant aux deux lypes ilaliques recomnus dans le gisement sous-marin de Marscille ${ }^{2 b t s}$ :

amphore ilalique (type I de l)ressel), à col élevé et lèvre droile ou concave avee gorge (haul: $\left.0^{\mathrm{m}}, 04\right)$, eir pâle rouge, Irès cuile avee granulations noirâtres; un col avec marque en relief $\mathrm{CL} \cdot \mathrm{F} \mathrm{E}$, dans cachet rectangulaire $\left(0^{\mathrm{m}},\left(124 \times 0^{\mathrm{m}}, 13\right)\right.$;

amphore gréco-ilalique, à panse en forme de loupie el épaulement, lèvre inclinée parfois très étroile $\left(0^{\mathrm{m}}, 02\right)$, pied terminé par un empattement, en pâte allant du jaunâtre au rouge, avec incrustation de dégraissants el de tuileaux rouges; un bas de panse avec marque en relief $\mathrm{T}$ (haut.: $\left.0^{\mathrm{m}}, 012\right)$, dans cachet circulaire en creux de $0^{\mathrm{m}}, 022$ diam., analogue à une marque du Cayla de Mailhac ${ }^{3}$ et tris voisin d'un cachel circulajre d'une amphore de Roquepertuse.

Le mur E. de cette salle repose sur de larges substructions qui se retournent à angle droit et délimitent une seconde salle, dont le niveau plus élevé

(2) Gi. Fr. Winter et E. Pennice, Die hellenisliche Kunst in Pompei (Pavim. und figul. mos.), Berlin, 1938.

(2bis) Cf. F. Bewort, Amphores el céramiques de l'épate de Marseille, in Gallia, XII-1954, 1 , 1). 3\%, lig. $2,1 \mathrm{~ns}$ III el $1 \mathrm{~V}$.

(3) 0. Tafranle, Gallia, V, 1947, p. 114, fig. 24. 
est marqué par une épaisse couche de cendres avee ossements d'animaux, céramique campanienne, fragnents d'amphores et boulets de lithobole romain.

Ies deux édifices, d'un appareil différent de celui des insulae précédemment fouillées, révèlent une reconstruction hûtive, dans laquelle sont entrés des remplois d'éléments d'architecture de grande proportion, don! on retrouve les fragments dans le rudus de la rue. Ils se sont insérés dans le quadrillage d'insulae, dont les ruelles transversales (îlot XVI) ont été coupées par le nouvel édifice (Gallia, XI, 1953, p. 106).

L'ćlargissement de la i'ue exhaussée, qui a comblé les trous de poteau de la première époque, formant une sorte de place de plus de $8 \mathrm{~m}$ de largeur. semble indiquer que ces édifices avaient une importance religicuse; celle-ci est attestíe par la découverte d'une quinzaine de crûnes au niveau du sol de la salle hypostryle el de la voie, parliculièrement au pied des piliers remployés, - découverte qui confirme la datation préromaine de cet ensemble. Les crânes, qui ont subi l'action des eaux d'infiltration, sont en mauvais état et incomplets; aucun ossement ni aucune vertèbre cervicale n'ont été trouvés avec eux. L'identification de huit d'entre eux montre qu'ils appartenaient à des individus d'âge différent dont six ayant dépassé 40 ans, d'après le degré d'ossification des sutures de la calotle (table du Professeur Vallois) ${ }^{4}$. Trois portent des trous de percement pour l'enclouage ou la suspension, vers le haut du crâne, sur le frontal gauche. L'un, peut-être de femme, âgé de 50 ans, est percé d'un trou carré de $0^{\mathrm{m}}, 04$ de côté avec traces de rouille; le clou de fer (long. $\left.0^{\mathrm{m}}, 225\right)$, à tête convexe (diam. $\left.0^{\mathrm{m}}, 035\right)$ a été retrouvé dans le cràne (fig. 7). L'autre appartient à un homme de 30 à 40 ans; le trou, en moins bon chtat, présente ígalement des lraces de rouille. Des fragments d'autres calotles paraissent avoir été percés de la même façon. Un autre révèle un mode de suspension différent : le côté gauche du frontal, seul retrouvé, porte vers le milieu deux petits trous circulaires de $0^{\mathrm{m}}, 005$ de diam., distants de $0^{\mathrm{m}}, 10$, analogues aux trous de suspension des céramiques pour le passage d'une attache. Leur indice, dolichocéphale, est sensiblement différent des crànes des lombes grecques de la nécropole de Marseille et d'Ampurias.

Ce rite est connu par plusieurs exemples, qui révèlent une signification diffírente, mais également religieuse : le crûne cncloué du Puig Castelar, oppidum de la région de Barenlone, trouvé au pied du rempart", comme les squeletles el lo erâne d'enfant dépossés face à l'ennent dans des caissons à l'inté-

(4) L'élude a élé confiée à M. R. Chardes el au prof'esseur OLdivier, qui avec l'assistance du Dr. Lubert, étudie le mode d'enclouage du crâne.

(5) Crâne au musée de Barcelone, percé d'un trou rond, légèrement écaillé, en haut du crâne, à l'extrémité postérieure du frontal, cỏté droit, clou à tête carréc : Ampurias, IV, 1942, p. 103. 
rieur du rempart de l'oppidum de l'Impernal à Luzech ${ }^{6}$, du milieu du $\mathrm{II}^{\mathrm{e}}$ siècle av. J.-G., ont une valeur apotropaïquc. L'enclouage du crâne, dans les sépultures signalées dans le Nord de l'Espagne, à l'époque antique ${ }^{7}$, et en Gaule,

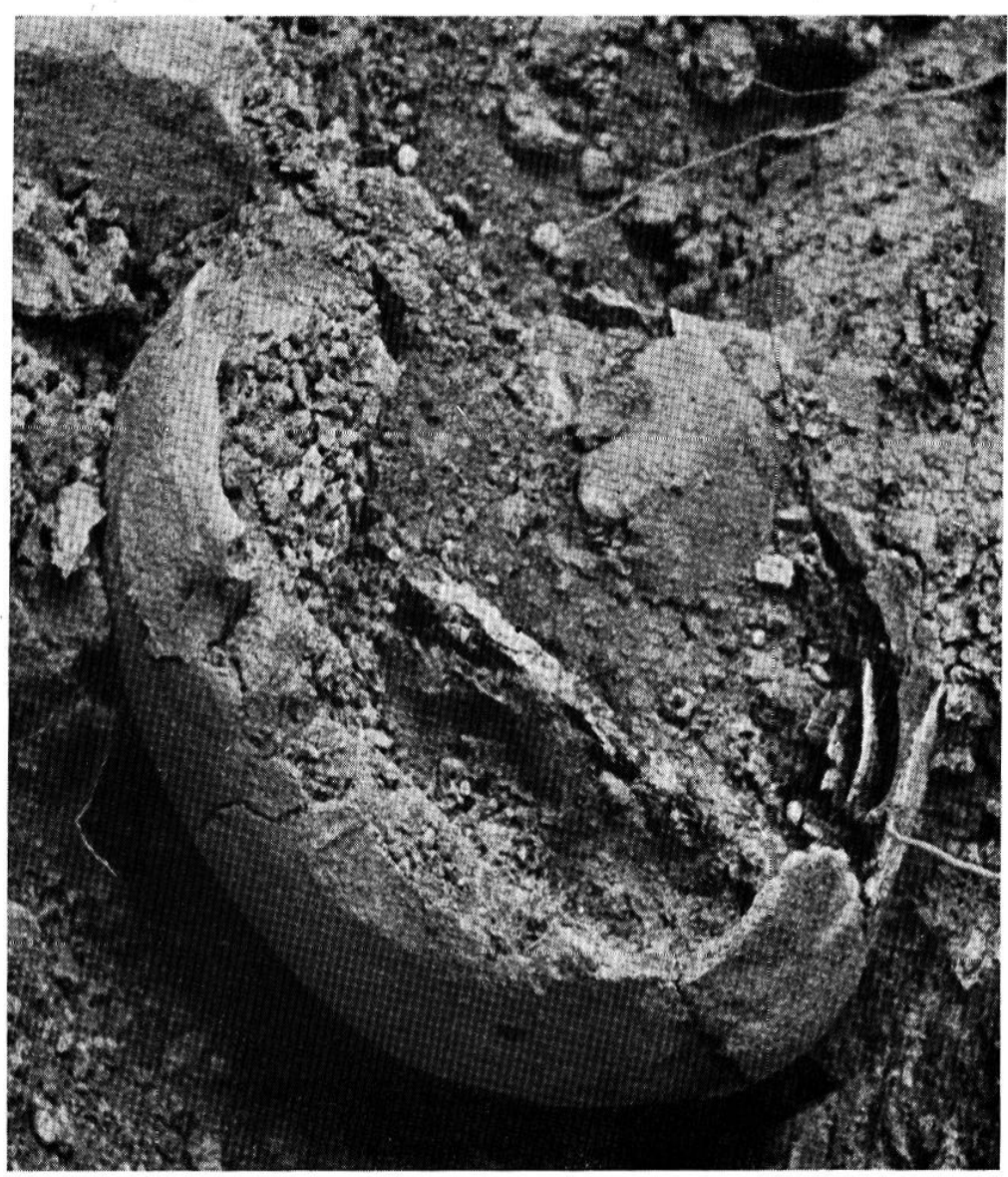

Cliché II. Ely.

FIG. 7. - Crâne enclouś d'un individı de 50 ans trouvé sur le sol la Voie Sacrée.

du Bas-Empire à l'époque barbare ${ }^{8}$, paraît obéir à la crainte superstitieuse du mort et à la peur du vampire. L'âge relativement avancé de certains de ces crânes, déjà noté à Roquepertuse ", l'emplacement dı local où étaient conser-

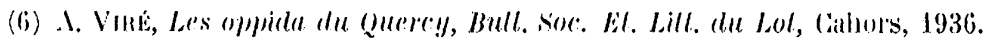

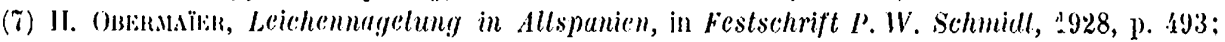
cité par R. LaNTEER, Bull, arch., 1943-45, p. 395.

(8) Ad. Blanchet et R. Lantier, Crânes percés de clous, ibid., p. 391.

(9) Quatre fragments identifiables appartenaient à des sujets jeunes et robustes, âgés de 30 à 40 ans, de 35 à 40 et de 35 à 45 : H. de GérIN-RICARd, Le sanctuaire préromain de Roquepertuse (Centenaire Soc. Stat., Marseille, 1927), p. 23. 
vés ces "trophées", associés sur un linteau avee une effigie sculptée, au centre de la ville, apportent un d́óment nouveau pour la signifieation religieuse du "culte du crîne", dont la fixalion avait pour fin de conserver l'esprit du défunt.

Crâne d'ennemi décapilé, dont la force vitale avait été mise au service de son vainqueur, ou relique des ancêtres ? L'enclouage, qui paraît avoir clé fait sur une têle fraîchement séparée du tronc, done avant décharnement et probablement momifiée, illustrerait ainsi, dans ce cas particulier, la description de la coulume barbare des Salyens, qui nous a été rapportéc par I)iodore de Sicile cl Strabon.

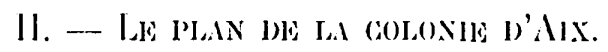

Trois déconvertes permellent de préciser le périmètre de la colonie el l'orientalion du cardo:

1) Le rempart occidental, précédemment signalé ${ }^{10}$, a été relrouvé plus au Sud, dans le clos Notre-Dame-de-la-Seds, à l'O. de la route d'Avignon. Il a ainsi été reconnu sur une longueur de $110 \mathrm{~m}$ environ; arasé vers le N. du clos, où il est à peu de profondeur, il est, au contraire, mieux conservé (fig. 8) vers le milieu jusqu'au Sud, où il a plus d'1 $m$ de hauteur ( 7 à 8 assises, dont deux pour le socle). D'une largeur de $2^{\mathrm{m}}, 20$, il présente un petit appareil smillé, régulier (moellons de $0^{\mathrm{m}}, 10 / 20$ long. et $0^{\mathrm{m}}, 08 / 14$ haul.), reposant sur IIn socle en forte saillie $\left(0^{\mathrm{m}}, 12\right)$. Au Sud, il faisait retour vers l'E., en direction de la "Porte d'Italie", par où pénétrail la voie romaine venant de Fréjus (la porte monumentale, en demi-lune, a été arasée à la fin du xvin siècle); le tracé du fronl sud ćlail encore visible, au débul du xixe siècle, sous la traverse de l'Aigle d'Or (rue Irma Moreau) ${ }^{11}$.

La délimitation vers l'Est et le Sud de la colonie romaine, à l'époque d'Auguste, permet ainsi de réviser le plan d'Aix qui se rapproche, par le périmètre de son enceinte (env. $4.000 \mathrm{~m}$ ) des grandes cilís augusliennes de la vallée du Rhône el de la Saône (fig. 9) ${ }^{12}$. Son arasemenl à une dale ancienne, sans doule par suite des troubles politiques du ve sicele, fut si radical, que son alignement disparaîl'a dans le tracó de's chemins de ce quartier, revenu à l'élat agreste, au Moỵen Age. Il se peupla d'habitations grossièrement établies sur des ruines, au Bas Empire. I. sol d'une casc, avee bélon de chaux assis sur la tranche arasée du rempart, au Sud de la fouille, a donné un petit

(10) Gillia, X1-1953, 1. 107.

(II) F.O.R., V, p. T3.

(12) Ci. Gallia, V-194i, p. 98 ; VI-1948, p. 209. 


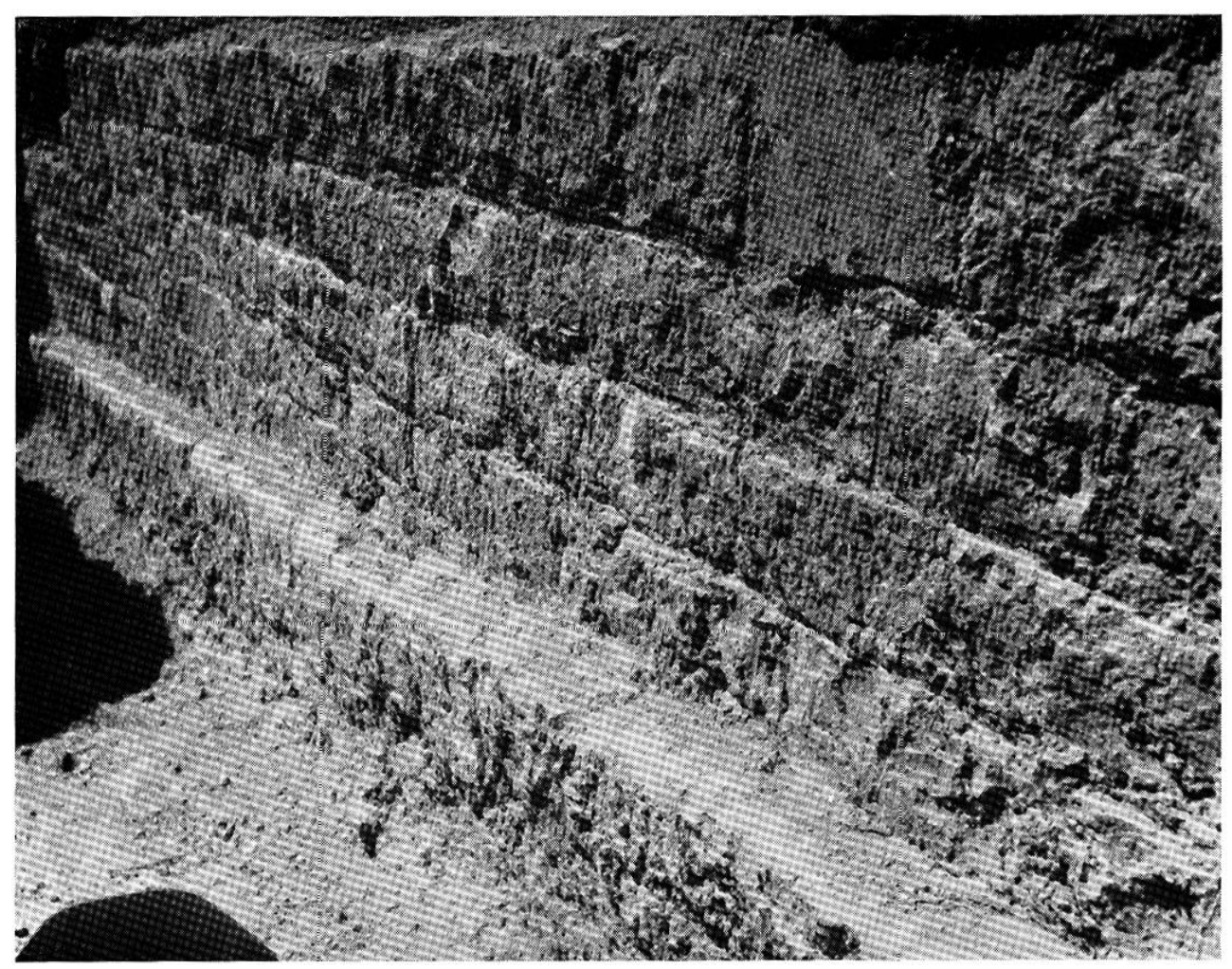

Cliche H. Ely.

Fic. 8. -.- Aix-en-Provence. Parement intérieur du rempart Ouest de la colonie.

bronze, lrès usé, de Conslance II (†361). A $55 \mathrm{~m}$ à l'E., des sondages onl mis au jour, a fleur de lerre, des restes de construction au sol bétonné, avee canalisation de pierre. Nombreux tessons de céramique grise estampée, dite

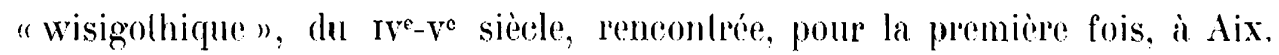

L'habilation de ec "quartier chrélien " entraina la rlisparition de grands édifices, et sans doule de l'amphithéatre, dont les arcades élaient visibles au dóbul du $x_{\text {vir }}$ siècle. Il avail servi de carrière de pierre jusqu'au moyen àge. Ite grands parpaings, régulièrement taillés $\left(1^{\mathrm{m}}, 30 \times 0^{\mathrm{m}}, 60 \times 0^{\mathrm{m}}, 41\right)$ avec trous de scellement, parfois avec mouluration, ont été trouvés aux abords de la parlie Sud du rempart, quelques-uns en remploi. A l'Est, près de la canalisation ci-dessus signalée, était, en remploi, dans un mur, une pierre, en grand appareil, sculptće sur une face de peltes el de boucliers, ayant appartenu, sans doule, à un arc de triomphe; il faut en rapprocher deux grands blocs, un voussoir orné d'un simpulum et un fragment d'entablement avec cuirasses, boucliers et glaives, trouvés en 1843, dans le même enclos, lors des fouilles de Rouard, à quelque $100 \mathrm{~m}$ au S.-E. ${ }^{13}$.

(13) ESPÉraNdiev, Recueil Bas-Reliefs, I, 100 et 101; F.O.R., V, p. 72. 


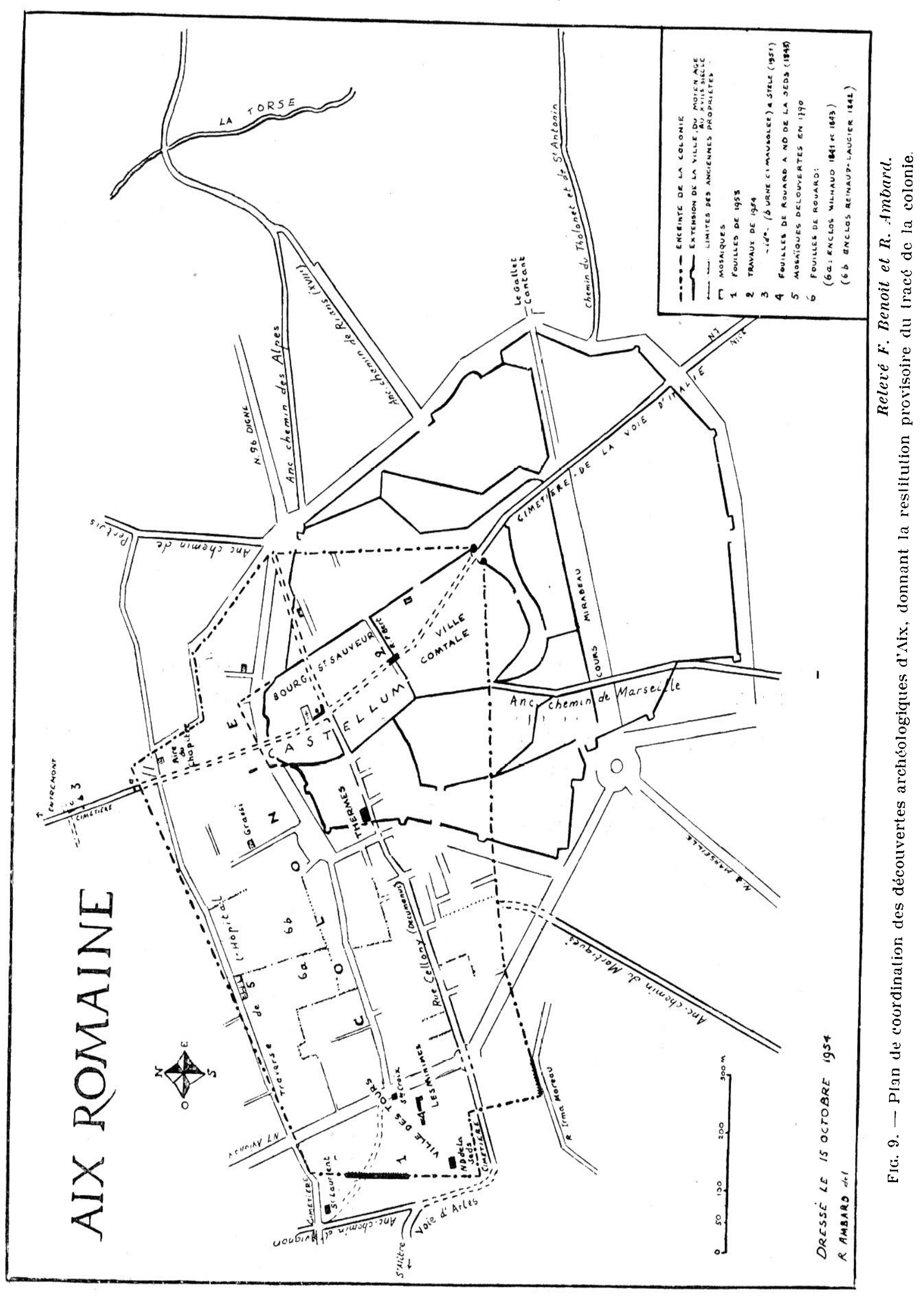


La continualion de la même ranchée a permis d'extraire d'un mur un aulel volif, en pierre de Bibemus (fig. 10), de basse ́́poque, creusé d'une cavité circulaire peu profonde pour les libations, a sa face supérieure, et représenlant, debout dans une niche rectangulaire, une Abondance ou une Déesse-mère, drapée, le voile relevé sur la tête au-dessus d'une couronne de cheveux; elle lient, de la main gauche, une corne d'abondance et présente, de la droite abaissée, un attribut indéterminé, sans doute une palère ${ }^{14}$.

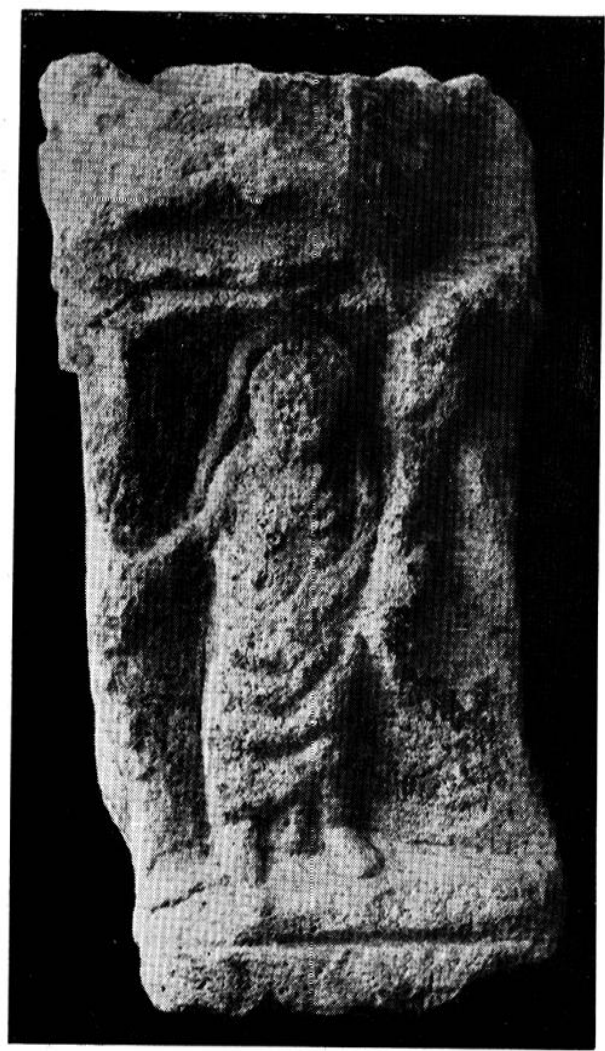

Cliché H. Ely.

FiG. 10, - Autel votif (haut. : o $0^{\mathrm{m}}, 20 ;$ ep. : $\left.0^{\mathrm{m}}, 15\right)$, remployé dans le « quarlier c'urélion 》 d'lix, pierre de Bibemus.
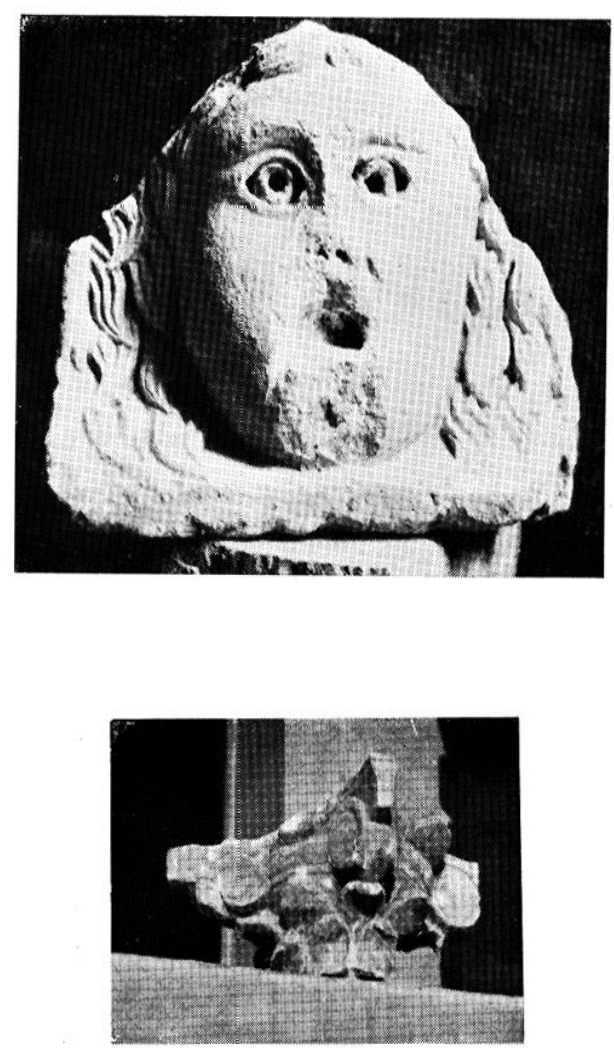

Fici. 11 (cn hautị. - Masque scénique. Acrolère, en pierre (haut: : $0^{\mathrm{m}}, 50 ;$ larg. : $0^{\mathrm{m}}, 55$ : ép. : $0^{\mathrm{m}}, 32$ ).

Fig. 12 (en bas). - Chapileau du Bas-Empire, en picrre (haut. : $0^{\mathrm{m}}, 25$; larg. de l'abaque : $0^{\mathrm{m}}, 45$;,

A une époque plus lardive, le quarlier servil de cimetière. Adossées à l'intérieur du rempart, ont été trouvées deux sépullures sous tuiles, orientées N.-S.,

(14) Cr. at six meme la pose d'une slatue d'. Whondanes, en mathe, probablement de provenance locale (Espéra.turtev, III, 2483; F.O.R., V, p. 81) (coll. Sallier). 
dont l'une avait son colfrage fait de dalles de marbre en remplor et de deux fragments d'épitaphes :

$$
\text { ...(V)ol(tinia)/...vir }
$$

(haut. : $0^{\mathrm{m}}, 14 ;$ long. : $0^{\mathrm{m}}, 23 ;$ ép. : $0^{\mathrm{m}}, 06$; haut. des lettres : $1^{\text {re }} l .: 0^{\mathrm{m}}, 06$; $2^{\text {e }}$ l. : $\left.0^{\mathrm{m}}, 04\right)$, et une inscription du Bas Empire, datée du consulat d'Opilio :

$$
\text { ...Opeliones } v \ldots / \text {...n }
$$$$
\text { . }
$$

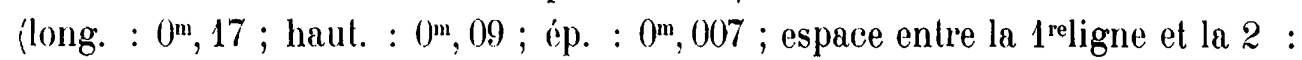
(0m, 08).

La date tardive de cette inscription, attestée par la substitution de l'E à l'I, et mentionnant le consulat d'Opilio, sans doute, en $524^{15}$, concorde avec celle des épitaphes chrétiennes datées qui viennent de ce cimetière : l'évêque Basilius, élu lors du rétablissement de l'évêché, en 475 († 494), Adjutor et Destrianus (début vi ${ }^{\mathrm{e}}$ siècle) ${ }^{\mathbf{1 6}}$.

C'est également à un remploi, qu'il faut attribuer un masque scénique de pierre, formant acrotère, assis sur une tablette (fig. 11), trouvé $\grave{a}-1^{\mathrm{m}}, 50$, sous la route de Berre, à hauteur de la conciergerie, près l'entrée de la chapelle de N.-D.-de-la-Seds, à quelques mètres à l'Est du passage supposé du rempart. L'expression singulière de la tête juvénile, encadrée de boucles en "virgule ", la bouche et les yeux, profondément creusís d'une cavité circulaire, permet de rapprocher cet acrotère d'un masque de marbre également aixois, aujourd'hui au musćc d'Avignon ${ }^{17}$, d'un acrotère de tombeau de Marseille et de celui de Glanum $^{18}$, à l'expression volontairement effrayante. La pupille profondément creusée, est entourée de l'annelet circulaire en relief de l'iris, lui-même détouré dans le globe de l'œil : cette technique est caractéristique des relief's du Bas-Empire, en Provence (lête du Lubéron ${ }^{19}$, stèle à portrait de Zosime, au musée Borély ${ }^{20}$, et se retrouve dans l'un des acrotères de Chorey, en Bourgogne ${ }^{21}$. Jécoration de tombeau, ayant une fonction apotropaïque, analogue à celle des têtes de Méduse.

Ainsi apparaît l'histoire de ce quartier, la "Ville des Tours" ou ville infórieure, appartenant à l'ćvêque au moyen âge, indépendante du castrum éta-

(15) Cf. les inscriptions d'Opilione et Vpilione cons. (524), à Vienne, p. c. Opilionis à Lyon (454 ou 525), peut-être Opilio, d Arles : LE BLANT, Inscrip. chrét. Gaule, nos $435,694,57$, cf. 695 et Suppl., $\mathrm{n}^{\circ} 106$.

(16) C.I.L., XII, 591, 590 el $592 ;$ F.O.R., V, p. 76, 1 à 3.

(17) Espérandieu, III, 2498; F.O.R., V, p. 80 (coll. Sallier).

(18) Espérandivu, I, 109; XII, 7861.

(19) Ibid., III, 2556.

(20) Ibid., I, 75. Gf. sur cette technique F. BENorT, Sculptures d'art provincial au Bas tmpire, en Provence, Provence Ilistorique, I, 1950, p. 20.

(21) Gallia, V-1947, p. 430, fig. 3. 
bli à l'Est de la colonie, à l'emplacement de l'ancien castellum de G. Sextius Calvinus ; quartier de grands édifices publics sous le Haut Empire, ruinés sous le Bas Empire, où fut, sans doute, transférée l'église épiscopale entourée de son cimetière, au ve s. et qui fut à nouveau abandonnée, au haut moyen ûge.

Au Nord, les travaux de construction du lolissement des Castors, à peu de profondeur, au dehors de l'enceinte, ont exhumé un petit chapiteau de pierre, de style composite, avec deux rangs de feuillage épannelé et des volutes angulaires, dont la spirale est remplacée par un cercle incisé, du Ive siècle (fig. 12).

2) L'établissement d'un réseau électrique souterrain, dans la rue Paul Bert (ancienne rue Saint-Laurent) a mis au jour une voie dallée, $\grave{a}-1 \mathrm{~m}$ sous la rue, au N.-E. du beffroi de l'Hôtel-de-Ville. La voie, pavée de grandes dalles $\mathrm{d}^{\prime} 1^{\mathrm{m}}, 50$ à $2 \mathrm{~m}$ de long., soigneusement juxtaposées avec joinls obliques et nercées de trous de louve, mesure $5^{\mathrm{m}}, 75$ de largeur; elle est comprise entre deux trottoirs (haut.: $\left.0^{\mathrm{m}}, 15\right)$, dont ne subsiste qu'un fragment de l'élément de l'Ouest. Le bord extérieur de la chaussée avait été entaillé par l'assise des trottoirs (fig. 13).

La direction de la voie S.E.-N.0., dans l'alignement de la "Porte d'Italie " au Sud, et vers le Nord de la façade Ouest de l'édifice romain de la cathédrale, permet de l'identifier au cardo de la colonie. Un segment de cette voie avait été signalé dans les caves des immeubles du côté Est de la rue Gaston de Saporla, dont l'alignement avait empiété sur la voie romaine ${ }^{22}$. Les mêmes travaux ont mis au jour, sur le côté Sud de la rue Paul Bert, à l'Est de cetle voie et au-dessous de son niveau, un mur en grand appareil, avec bossage, orienté E.-0., qui correspond, peut-être, à l'enceinte du castellum de 122.

3) L'alignement de cetle voie, qui paraît s'infléchir vers l'Est, après sa rencontre avec le decumanus (rues Cellony et du Bon Pasteur), a donné son orientation au réseau d'anciens chemins et aux limites de propriétés du quartier nord d'Aix, non repeuplé au Moyen Age (Ville des Tours). L'ouverture d'un collecteur, route de Puyricard, a permis de retrouver cet alignement par le dégagement du stylobate occidental (long. : $4^{\mathrm{m}}, 15$ ), en grand appareil, d'un édifice tétrapyle, probablement un mausolée, $\grave{a}-1^{\mathrm{m}}, 25$ sous le niveau de la route. Des déblais a été retiré l'angle d'un petit fronton de pierre, orné de denticules (long.: $0^{\mathrm{m}}, 34$; haut.: $0^{\mathrm{m}}, 34$ ) avec départ d'acrotère à palmetles.

Au Sud, ont été mis au jour deux coffrages de pierre, dont l'un contenait une urne cinéraire de marbre, de forme circulaire (tessère anépigraphe) avec

(22) M. Cuenc, Aquae Sextiae, 1916, p. 367. 


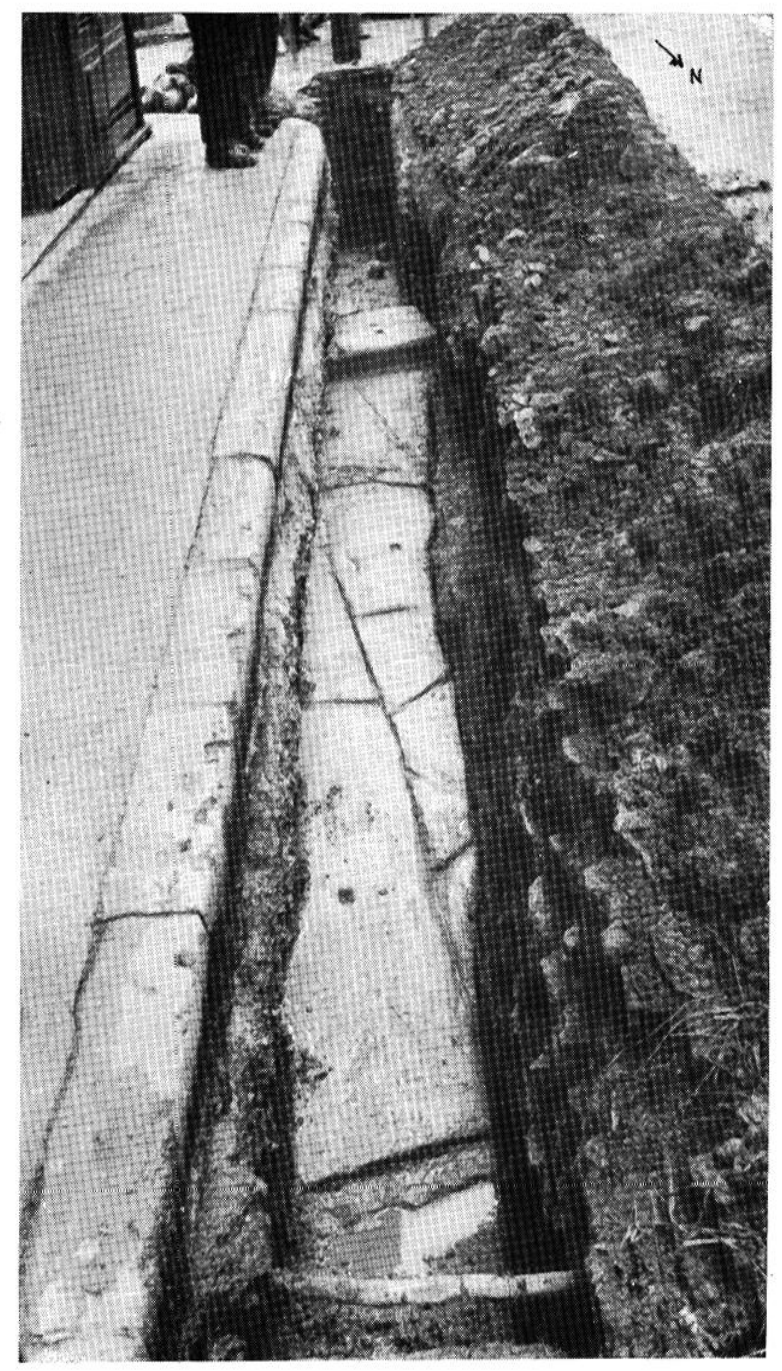

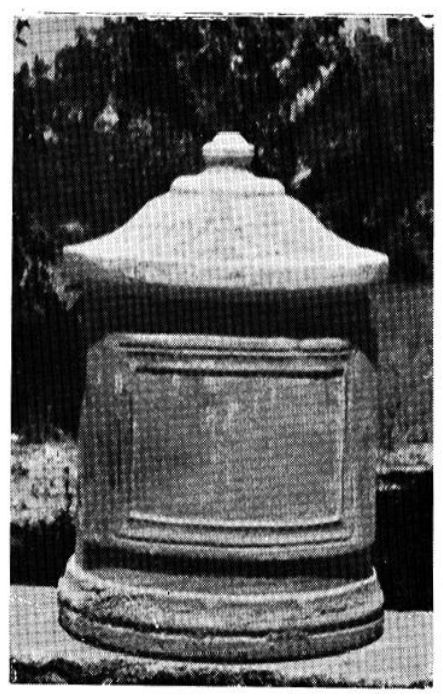

Cliche t. Gomorest.

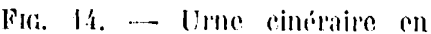
mantre (baul. avec te couverele : $0^{m}, 10$; diam. : (1)"m,23) du cimelière de la roule de Puyricard.

Fra. 13. - Dallage du cardo de la colonic, rue Paul-Bert.

Cliché II. Ely.

son couvercle orné d'imbricalions (fig. 14); ì l'inlírieur, ecnders calcinées, sans objet mobilier' (haut.: $0^{\mathrm{m}}, 40$; diam.: $\left(0^{\mathrm{m}}, 25\right)$.

Cé cimelière était adossé à l'Est à la colline rocheuse; une découverte antérieure ${ }^{23}$, sur la même route, en face la chapelle de l'Hôpital, à $100 \mathrm{~m}$ environ au Sud, permet de préciser son périmètre. 11 bordait la voie des Alpes, au sorlir de la porte Nord de la colonie, situcé a hauleur de la traverso de l'Jlôpital sans qu'il soit possible cneore de situer de faģon précise l'alignement Nord de l'enceinte (fig. 9).

Fernand Benoît'. 